\title{
Ideal cardiovascular health and psychosocial risk factors among Finnish female municipal workers
}

\section{Veromaa, Veera}

2017-02

Veromaa , V , Kautiainen, H , Saxen , U , Malmberg-Ceder, K, Bergman, E \& Korhonen, P E 2017 , ' Ideal cardiovascular health and psychosocial risk factors among Finnish female municipal workers ' , Scandinavian Journal of Public Health , vol. 45 , no. 1 , pp. 50-56 . https://doi.org/10.1177/1403

http://hdl.handle.net/10138/232262

https://doi.org/10.1177/1403494816677661

publishedVersion

Downloaded from Helda, University of Helsinki institutional repository.

This is an electronic reprint of the original article.

This reprint may differ from the original in pagination and typographic detail.

Please cite the original version. 


\title{
Ideal cardiovascular health and psychosocial risk factors among Finnish female municipal workers
}

\author{
VEERA VEROMAA ${ }^{1,2}$, HANNU KAUTIAINEN ${ }^{3,4,5}$, ULLA SAXEN ${ }^{6}$, \\ KIRSI MALMBERG-CEDER ${ }^{7}$, ELINA BERGMAN ${ }^{1}$ \& PÄIVI E. KORHONEN ${ }^{1,2}$
}

\begin{abstract}
${ }^{1}$ Institute of Clinical Medicine, Family Medicine, University of Turku and Turku University Hospital, Finland, ${ }^{2}$ Central Satakunta Health Federation of Municipalities, Finland, ${ }^{3}$ Department of General Practice and Primary Health Care, University of Helsinki and Helsinki University Hospital, Helsinki, Finland, ${ }^{4}$ Folkhälsan Research Center, Helsinki, Finland O) Unit of Primary Health Care, Kuopio University Hospital, Kuopio, Finland, ${ }^{5}$ Unit of Primary Health Care, Kuopio University Hospital, Kuopio, Finland, ${ }^{6}$ Department of Psychiatry, Hospital District of Satakunta, Finland, ${ }^{7}$ Institute of Clinical Medicine, Department of Neurology, University of Turku and Turku, University Hospital, Finland
\end{abstract}

\begin{abstract}
Aims: Ideal cardiovascular health has been defined by the American Heart Association as the absence of disease and the presence of seven key health factors and behaviours. However, little is known about the mental aspects associated with ideal cardiovascular health metrics. The objective of this study was to assess the relationships between psychosocial risk factors and ideal cardiovascular health metrics among Finnish women at municipal work units. Method: A cross-sectional study was conducted in Finland among 732 female employees (mean \pm SD age $48 \pm 10$ years) from ten work units in 2014 . Ideal cardiovascular health metrics were evaluated with a physical examination, laboratory tests, medical history and selfadministrated questionnaires. Psychosocial risk factors (social isolation, stress, depressive symptoms, anxiety, hostility and type D personality) were assessed with core questions as suggested by the European Society of Cardiology. Results: The prevalence of having 5-7 ideal cardiovascular health metrics was $183(25.0 \%)$, of whom $54.1 \%$ had at least one psychosocial risk factor. Anxiety (31.3\%), work stress (30.7\%) and type D personality $(26.1 \%)$ were the most prevalent of the psychosocial risk factors. The prevalence of depressive symptoms $(p<0.001)$ and type D personality $(p=0.049)$ decreased linearly according to the sum of ideal cardiovascular health metrics after adjustment for age and years of education. Conclusions: Even women with good cardiovascular health are affected by psychosocial risk factors at municipal work units. Although the association is possibly bidirectional, screening and treating depression and dealing with type D personality might be crucial in improving cardiovascular health among women.
\end{abstract}

Key Words: Ideal cardiovascular health, psychosocial risk factors, depression, type D personality, women

\section{Introduction}

Several population-based studies in Europe and the USA have shown that coronary event rates have increased or plateaued during the past two decades in young and middle-aged women [1-3]. This trend is probably explained by ongoing unhealthy behaviours such as physical inactivity and adverse dietary habits, which have led to an increased prevalence of obesity and type 2 diabetes $[4,5]$.
To reverse the rising tide of cardiovascular disease (CVD), the American Heart Association (AHA) has adopted the concept of ideal cardiovascular health $(\mathrm{CVH})$, defined by the absence of clinically manifest CVD together with the presence of both ideal health behaviours (non-smoking, normal body mass index, physical activity at goal, healthy diet) and ideal health factors (untreated total cholesterol $<5.18 \mathrm{mmol} / \mathrm{l}$,

Correspondence: Veera Veromaa, Institute of Clinical Medicine, Family Medicine, University of Turku and Turku University Hospital, Turku, Finland. E-mail: vimver@utu.fi

(Accepted 4 October 2016)

(C) Author(s) 2016

Reprints and permissions: sagepub.co.uk/journalsPermissions.nav

DOI: $10.1177 / 1403494816677661$

sjp.sagepub.com

(SAGE 
untreated blood pressure $<120 \mathrm{mmHg}$ systolic and $<80 \mathrm{mmHg}$ diastolic, fasting plasma glucose $<5.55$ mmol/l) [6]. This metric, referred to as Life's Simple 7 , is a method of assessing the $\mathrm{CVH}$ of a population and has the aim of reducing CVD mortality and improving CV risk factors in the US population by $20 \%$ by 2020 [6]. However, ideal CVH is difficult to achieve. Only $0.1 \%$ of the US adult population fulfil all seven of the AHA's ideal health metrics [7]. In Finland, only $9 \%$ of women and $3 \%$ of men aged 25 74 years have $\geqslant 5$ ideal health metrics [8], although rates of coronary heart disease in working-aged men have decreased by $80 \%$ since the late 1960 s [9].

Psychosocial risk factors act as barriers to the adoption of a healthy lifestyle and have a tendency to accumulate. Low socioeconomic status, lack of social support, stress, depression, anxiety, hostility and a type $\mathrm{D}$ personality also contribute to the risk of developing CVD and the worsening of the clinical course and prognosis of CVD [10,11]. The European 2012 guidelines on CVD prevention in clinical practice recommended the evaluation of psychosocial risk factors with core questions. The guidelines indicate that there are gaps in the evidence for CVD prevention in women and young adults [11]. The aim of the present study was to assess the relationships with psychosocial risk factors using Life's Simple 7 among Finnish women at municipal work units. We hypothesized that psychosocial risk factors might have an effect in reaching ideal CVH.

\section{Materials and methods}

\section{Participants}

PORi To Aid Against Threats is a longitudinal study conducted among employees of the city of Pori $(83,497$ inhabitants in 2014) in southwestern Finland. The study population consisted of workers from 10 work units selected by the chief of the welfare unit of Pori. Invitation and study information letters were sent to the employees as an email attachment by the managers of the work units. We cannot report the exact participation rate for the study because some employees may have ignored the invitation and information letter sent by email. Information events were also organized for employees. There was no exclusion criterion. In total, 836 employees (104 men, 732 women) consented to participate in the study. Because of the overwhelming predominance of women, which corresponds to the sex distribution of the Pori employees, men were excluded from the study cohort. Respondents from libraries $(n=22)$, museums $(n=33)$, technical management $(n=80)$, social services $(n=196)$ and health care units $(n=401)$ were invited to an enrolment appointment with the study nurse. Occupations included librarians, museum employees, groundkeepers, computer workers, social workers, nurses, physicians, administrative officials and general office staff. The present cross-sectional study consisted of white, female employees (age range 19-66 years) who completed self-administrated questionnaires at home before the baseline examination.

\section{Measures}

Ideal cardiovascular health metrics. Smoking status was assessed by a questionnaire. Non-smoking was defined as having never smoked or to have stopped smoking >12 months ago. Height and weight were measured by a study nurse with the participants in a standing position without shoes and outer garments. Weight was measured to the nearest $0.1 \mathrm{~kg}$ with calibrated scales and height to the nearest $0.5 \mathrm{~cm}$ with a wall-mounted stadiometer. Body mass index (BMI) was calculated as weight $(\mathrm{kg})$ divided by the square of height $\left(\mathrm{m}^{2}\right)$. The ideal BMI was $<25.0 \mathrm{~kg} / \mathrm{m}^{2}$.

Physical activity was assessed using a questionnaire that asked the frequency and duration of leisure time and commuting activities in a typical week. Physical activity at goal was defined as engaging in $\geqslant 150$ minutes per week of moderate intensity activities or $\geqslant 150$ minutes per week of moderate + vigorous intensity activities [6].

Information of diet was collected with a food-frequency questionnaire. Daily consumption of fruits, vegetables, whole grains, unsaturated dietary fats and white meat (poultry, fish) at least three times a week was considered as a healthy diet. Intake of the ideal level of each dietary component was scored with one point for a range of $0-5$. The dietary $\mathrm{CVH}$ metric was categorized as ideal if a dietary score of 4-5 was achieved, as suggested in the AHA's recommendation of ideal metrics [6].

Blood pressure was measured by a study nurse with an automatic validated blood pressure monitor with the participants in a sitting posture after resting for at least 5 minutes. A larger cuff was used for participants with an arm circumference $>32 \mathrm{~cm}$. Two readings taken at intervals of at least 2 minutes were measured and the mean of these readings was used in the analysis.

Laboratory tests were carried out on blood samples obtained after at least 8 hours of fasting. Total cholesterol was measured enzymatically (Architect c4000/c8000). Glucose tolerance was measured with glycated haemoglobin (HbA1c), which was determined by high-performance liquid chromatography (Tosoh HLC-723G7 (G7)). The AHA metric uses 
fasting plasma glucose to determine normoglycaemia; however, we used $\mathrm{HbAlc}$ because it gives an indication of glycaemia over several preceding weeks rather than at a single time point [12]. Normoglycaemia was defined as $\mathrm{HbAlc}<6.0 \%$ ( $<42 \mathrm{mmol} / \mathrm{mol})$ [13].

The seven ideal $\mathrm{CVH}$ metrics were grouped into three categories of ideal CVH metrics $(0-2,3-4$ and 5-7). This classification is based on previous research of the AHA ideals, where this classification was used to describe poor (0-2 ideal CVH metrics), intermediate (3-4 CVH metrics) and ideal (5-7 CVH metrics) levels of cardiovascular health $[14,15]$.

Psychosocial risk factors. The study nurse assessed psychosocial risk factors in clinic by core questions suggested by the European 2012 Guidelines on CVD Prevention in Clinical Practice [11]:

- Work and family stress. Do you have enough control over how to meet the demands at work? Is your reward appropriate for your effort? Do you have serious problems with your spouse?

- Social isolation. Are you living alone? Do you lack a close confidant?

- Depression. Do you feel down, depressed and hopeless? Have you lost interest and pleasure in life?

- Anxiety. Do you frequently feel nervous, anxious, or on edge? Are you frequently unable to stop or control worrying?

- Hostility. Do you frequently feel angry over little things? Do you often feel annoyed about habits other people have?

- Type D personality. In general, do you often feel anxious, irritable, or depressed? Do you avoid sharing your thoughts and feelings with other people?

A 'yes' answer to one or more of these questions was indicated as a likely psychosocial risk factor.

Other measures. Using self-administrated questionnaires and medical records, information was gathered about diseases diagnosed by a physician, years of education, occupational status, marital status (cohabiting or not) and quality of sleep (good or not good). Financial satisfaction was assessed with the question 'I have to spare expenditures' (yes or no). Alcohol consumption was assessed with the three-item Alcohol Use Disorders Identification Test (AUDIT-C) with a cut-off value of 5 for harmful drinking [16].

\section{Informed consent}

The study protocol and consent forms were reviewed and approved by the ethics committee of the Hospital
District of Southwest Finland. All participants provided written informed consent for the project and subsequent medical research.

\section{Statistical analysis}

Statistical significance for the hypotheses of linearity was evaluated by using the Cochran-Armitage test and generalized linear models with appropriate distribution and link function. In the case of violation of the assumptions (non-normality), a bootstrap-type test was used. The normality of the variables was tested by using the Shapiro-Wilk W test. All analyses were performed using STATA 14.0 (StataCorp LP, College Station, TX, USA).

\section{Results}

We evaluated 732 female employees (mean \pm SD age $48 \pm 10$ years). The prevalence of having $5-7$ ideal $\mathrm{CVH}$ metrics was $183(25.0 \%)$, with $9(1.2 \%)$ women achieving all seven AHA criteria for ideal CVH. Table I shows the characteristics of the participants with $0-2,3-4$ or $5-7 \mathrm{CVH}$ metrics at the ideal level.

Participants with more ideal $\mathrm{CVH}$ metrics were more likely to be younger, more educated and financially more satisfied. They were also more likely to be cohabiting and had a better quality of sleep. The prevalence of diabetes and hypertension was lower with increasing levels of $\mathrm{CVH}$ metrics.

There were $272(37.2 \%)$ women who had none of the psychosocial risk factors, $166(22.7 \%)$ reported one, $205(28.0 \%)$ reported two to three and 89 $(12.2 \%)$ reported four to six psychosocial risk factors. Figure 1 shows the distribution of the sum of components of ideal $\mathrm{CVH}$ metrics and psychosocial risk factors.

Of the 272 women with none of the psychosocial risk factors, $84(30.9 \%)$ had 5-7 CVH metrics, 136 $(50.0 \%)$ had 3-4 metrics and $52(19.1 \%)$ had 0-2 metrics at the ideal level, respectively.

Non-smoking $(86.7 \%)$ and normoglycaemia (92.5\%) were the CVH metrics with the highest ideal prevalence, whereas untreated blood pressure $<120 / 80 \mathrm{mmHg}(18.9 \%)$ was the most infrequent. Anxiety (31.3\%), work and family stress (30.7\%) and type D personality $(26.1 \%)$ were the most prevalent of the psychosocial risk factors. The prevalence of hostility (20.8\%), depressive symptoms (18.7\%) and social isolation $(17.3 \%)$ were the most infrequent. There was notably less variation in the prevalence of different psychosocial risk factors than in the $\mathrm{CVH}$ metrics. (Figure 2).

Of the psychosocial risk factors, the prevalence of depressive symptoms and social isolation decreased 
Table I. Characteristics of study participants according to the sum of ideal cardiovascular health metrics.

\begin{tabular}{lcccc}
\hline Variable & \multicolumn{2}{l}{ Sum of ideal cardiovascular health metrics } & & \multirow{2}{*}{$p$ for linearity } \\
\cline { 2 - 5 } & $0-2(n=164)$ & $3-4(n=385)$ & $5-7(n=183)$ \\
\hline Age (years) & $52(8)$ & $48(10)$ & $44(10)$ & $<0.001$ \\
Education (years) & $13.4(2.7)$ & $13.9(2.7)$ & $14.5(2.6)$ & $<0.001$ \\
Financial satisfaction & $85(52)$ & $240(62)$ & $130(71)$ & $<0.001$ \\
Marital status, cohabiting & $116(71)$ & $296(77)$ & $153(84)$ & 0.005 \\
Good quality of sleep & $101(64)$ & $280(73)$ & $135(74)$ & 0.037 \\
AUDIT-C & $3.02(1.81)$ & $2.90(1.68)$ & $2.79(1.75)$ & 0.25 \\
Previously diagnosed comorbidities & & & $2(1)$ & $<0.001$ \\
Diabetes mellitus & $15(9)$ & $10(3)$ & $15(8)$ & 0.001 \\
Hypertension & $38(23)$ & $62(16)$ & $2(1)$ & 0.46 \\
Coronary heart disease & $1(1)$ & $1(1)$ & $8(4)$ & 0.44 \\
Depression & $4(2)$ & $25(6)$ & $5(3)$ & 0.78 \\
Asthma & $7(4)$ & $5(1)$ & $31(17)$ & 0.27 \\
Cancer & $1(1)$ & $57(15)$ & $9(5)$ & 0.81 \\
Musculoskeletal disorders & $27(16)$ & $27(7)$ & $7(4)$ & 0.023 \\
Gastrointestinal disorders & $15(9)$ & $39(10)$ & \\
Thyroidal disorders & $19(12)$ & &
\end{tabular}

Data presented as mean \pm SD values or $n(\%)$.

AUDIT-C: Alcohol Use Disorders Identification Test.

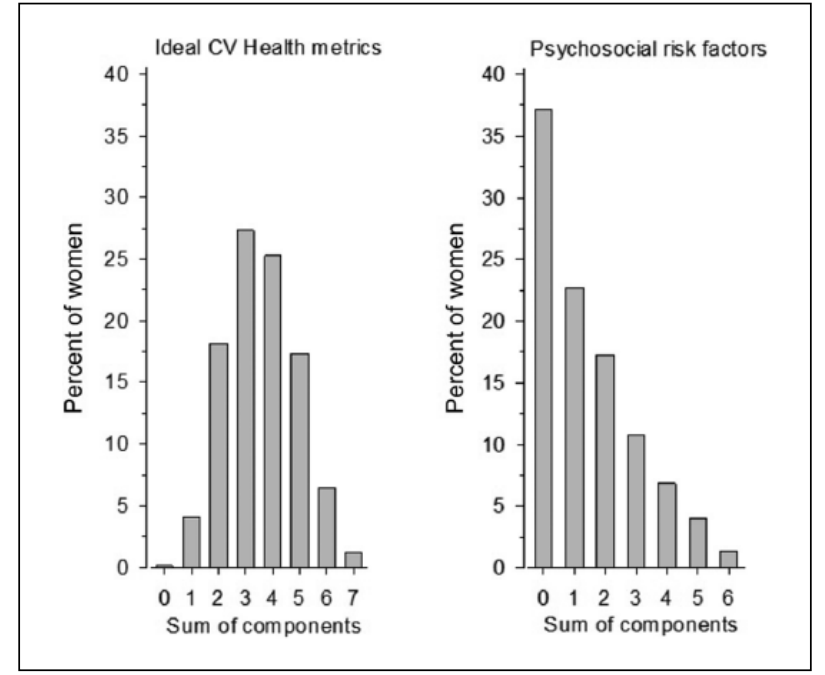

Figure 1. Distribution of the sum of components of ideal cardiovascular $(\mathrm{CV})$ health metrics and psychosocial risk factors.

linearly according to the sum of ideal CVH metrics. After adjustment for age and years of education, the association was statistically significant with depressive symptoms and type D personality, but slightly attenuated with social isolation. Even among women with 5-7 ideal CVH metrics, $54.1 \%$ had at least one psychosocial risk factor (Table II).

\section{Discussion}

The present study indicates that female employees with psychosocial risk factors are less likely to attain ideal CVH. The sum of ideal CVH metrics decreased linearly with depressive symptoms and type D personality. There was also a trend towards an inverse linear relationship between social isolation and the sum of ideal health metrics. Of our study participants (age range 19-66 years), 25\% had 5-7 CVH metrics at the ideal level. In a Spanish study, $19 \%$ of women aged $\geqslant 18$ years achieved $5-7$ ideal $\mathrm{CVH}$ metrics [17]. In our study, the proportion of ideal CVH metrics increased to $31 \%$ in women who had no psychosocial risk factor.

Low education level and low socioeconomic status have been shown to be associated with lower ideal CVH scores [18-20]. The prevalence of ideal levels of CVH metrics increased linearly with years of education and financial satisfaction in the present study, although we evaluated women who had been attended school in the Finnish nine-year compulsory education system and who currently had a stable workplace. Excluding low education and poor financial situation, almost two out of three women in our study had at least one of the six additional psychosocial risk factors that have been shown to contribute to the development and adverse outcome of coronary heart disease. This may partly explain why women are still dying prematurely of CVD.

There are only few studies reporting the relationships of psychosocial risk factors with ideal CVH. In a Chinese urban population, depression, anxiety and stress were negatively correlated with ideal CVH [21]. We found no association of anxiety or stress with ideal CVH, although one in three of our study participants reported these psychosocial risk factors. Results linking CVD and anxiety are incoherent, 


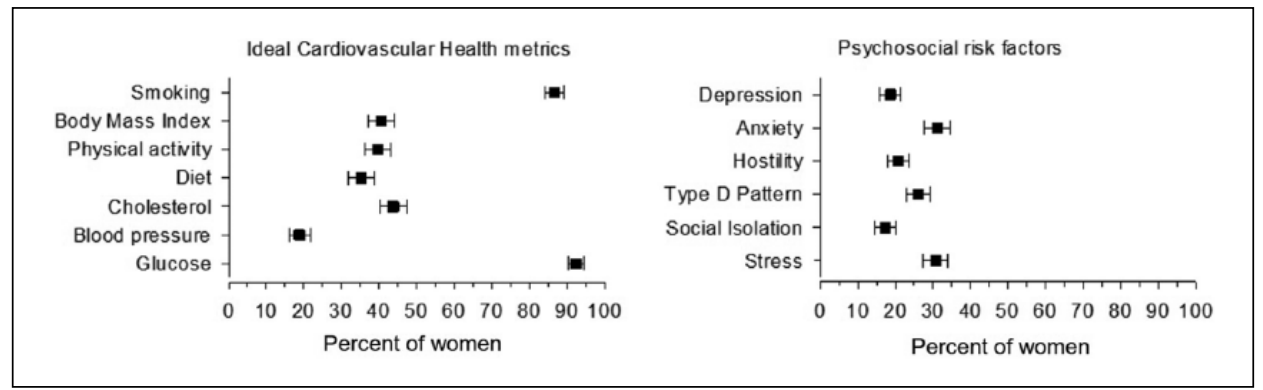

Figure 2. Prevalence of ideal cardiovascular health metrics and psychosocial risk factors among the female employees.

Cut-offs for ideal cardiovascular health metrics: smoking, never smoked or quit smoking $>12$ months ago; body mass index $<25.0 \mathrm{~kg} / \mathrm{m}^{2}$; physical activity, $\geqslant 150$ minutes per week of moderate intensity activities or $\geqslant 150$ minutes per week of moderate + vigorous intensity activities; diet, four to five ideal healthy diet components; cholesterol, untreated total cholesterol $<5.18 \mathrm{mmol} / 1$; blood pressure, untreated blood pressure $<120 / 80 \mathrm{mmHg}$; glucose, untreated $\mathrm{HbA} 1 \mathrm{c}<6.0 \%(<42 \mathrm{mmol} / \mathrm{mol})$.

Table II. Prevalence of psychosocial risk factors by the sum of ideal cardiovascular health metrics.

\begin{tabular}{|c|c|c|c|c|c|}
\hline \multirow[t]{2}{*}{ Psychosocial risk factors } & \multicolumn{3}{|c|}{ Sum of ideal cardiovascular health metrics } & \multicolumn{2}{|c|}{$p$ for linearity } \\
\hline & $0-2(n=164)$ & $3-4(n=385)$ & $5-7(n=183)$ & Crude & Adjusted $^{\mathrm{a}}$ \\
\hline Any psychosocial risk factor & $112(68)$ & $249(65)$ & $99(54)$ & 0.009 & 0.033 \\
\hline Depressive symptoms & $44(27)$ & $74(19)$ & $19(10)$ & $<0.001$ & $<0.001$ \\
\hline Anxiety & $53(32)$ & $126(33)$ & $49(27)$ & 0.32 & 0.31 \\
\hline Hostility & $33(20)$ & $85(22)$ & $34(19)$ & 0.80 & 0.97 \\
\hline Type D personality & $45(27)$ & $111(29)$ & $35(19)$ & 0.12 & 0.049 \\
\hline Social isolation & $36(22)$ & $70(18)$ & $21(11)$ & 0.013 & 0.059 \\
\hline Stress & $58(35)$ & $113(29)$ & $54(30)$ & 0.21 & 0.13 \\
\hline
\end{tabular}

Data presented as $n(\%)$.

aAdjusted for age and years of education.

changing from anxiety being a protective mechanism to a risk factor [22]. With regard to work-related stress, the association with the risk of CVD is also debatable [23]. We did not find a correlation between hostility and ideal CVH. This is new information about the connection, as we could not find any other study evaluating this association.

Of our study participants, $19 \%$ reported depressive symptoms. In the USA, the prevalence of depressive symptoms was $20 \%$ among all participants of the NHANES survey in 2005-2008 and 26\% among women [24]. In our study, $27 \%$ of women with $0-2$ ideal health metrics reported depressive symptoms. In the USA, an inverse relationship has been observed between depressive symptoms and ideal CVH metrics $[14,25]$. A recent French study gave a first-time insight to Europe, when they observed depressive symptoms as barriers to reaching ideal CVH [15]. In these studies, the prevalence of depressive symptoms changed from 7 to $11 \%[14-15,25]$. The variation in the study results may reflect different study populations and assessment methods for depressive symptoms, as well as cultural differences. Only $2 \%$ of the women in our study had previously diagnosed depression, but we evaluated participants active in working life. Depression is one of the single most disabling conditions [26] and thus people with depression may have already dropped out from working life or may have ignored the invitation to the study. This healthy worker effect may also emerge if people outside the workforce are not included in a study population.

This is the first study to report a negative association between ideal CVH and type D personality. ' $D$ ' stands for 'distressed' and this personality type has negative feelings (negative affectivity) and avoids social contact (social inhibition) [11]. In our study population, $26 \%$ were categorized as type $\mathrm{D}$ personalities. CVD patients with type D personality have a greater than three-fold risk for both CVD events and a poorer quality of life [27]. Recognizing personality patterns is important for customized patient education, even though it is not possible to change personality.

We found evidence of a decreasing sum of ideal $\mathrm{CVH}$ metrics with social isolation, although the test for linear association was not significant $(p=0.059)$. Cohabiting was associated with an increasing number of ideal CVH metrics. According to a large Danish study, there is a two-fold risk of acute 
coronary syndrome among single people [28]. The proportion of people living alone has increased substantially in developed countries, which may affect population health in previously unconsidered ways.

The major limitation of our study is its crosssectional nature, which prevented us assessing any causalities between the Life's Simple 7 and psychosocial risk factors. Thus the association between psychosocial risk factors and ideal $\mathrm{CVH}$ may be bidirectional. Self-reporting of diet, physical activity and smoking status may also be unreliable, but we tried to overcome this bias by using validated questionnaires and standardized procedures. We assessed psychosocial risk factors with core questions that can be used as a preliminary assessment within a clinical interview [11]. Giving an affirmative answer to either one of the two core questions on depression used in the present study has been shown to be as effective as using longer screening instruments [29].

The strengths of our study are that the wide-ranging data came from a representative sample of Finnish women working at municipal work units, which enabled us to find women-focused nuances of CVD prevention. Anthropological measurements were made by trained medical staff. We also could take into account many factors at work and in leisure time, which enabled us to analyse the association between psychosocial risk factors and ideal $\mathrm{CVH}$ metrics with good precision.

\section{Conclusions}

Psychosocial risk factors have a considerable relationship with ideal $\mathrm{CVH}$ metrics in women at municipal work units. Even among the women with 5-7 ideal $\mathrm{CVH}$ metrics, over $50 \%$ had at least one psychosocial risk factor. These psychological problems may be underdiagnosed and untreated. Although the association may be bidirectional, screening and treating depression and dealing with a type $\mathrm{D}$ personality and social isolation might be crucial in improving $\mathrm{CVH}$ among women of working age.

\section{Authorship}

VV, HK, KM and PK contributed to the conception or design of the work. VV, HK, US, EB and PK contributed to the acquisition, analysis, or interpretation of data for the work. VV, HK and PK drafted the manuscript. All authors critically revised the manuscript. All gave final approval and agree to be accountable for all aspects of work ensuring integrity and accuracy.

\section{Conflict of interest}

The authors declared no potential conflicts of interest with respect to the research, authorship, and/or publication of this article.

\section{Funding}

The authors disclosed receipt of the following financial support for the research, authorship, and/or publication of this article. This study was supported by the Hospital District of Satakunta, the Hospital District of Southwest Finland and Etera Mutual Pension Insurance Company. The funding sources had no role in the design and conduct of the study, collection, management, analysis and interpretation of the data, preparation, review, or approval of the manuscript and decision to submit the manuscript for publication.

\section{References}

[1] Towfighi A, Zheng L and Ovbiagele B. Sex-specific trends in midlife coronary heart disease risk and prevalence. Arch Intern Med 2009;169:1762-6.

[2] Lehto H, Lehto S, Havulinna A, et al. Are coronary event rates declining slower in women than in men - evidence from two population-based myocardial infarction registers in Finland? BMC Cardiovasc Disord. 2007;7:35.

[3] Mannsverk J, Wilsgaard T, Njølstad I, et al. Age and gender differences in incidence and case fatality trends for myocardial infarction: a 30-year follow-up. The Tromsø Study. Eur 7 Prev Cardiol 2012;19:927-34.

[4] Yang Q, Cogswell M, Flanders W, et al. Trends in cardiovascular health metrics and associations with all-cause and CVD mortality among US adults. $\mathcal{F} A M A$. 2012;307: 1273-83.

[5] Lehto H, Lehto S, Havulinna A, et al. Gender differences in the prevalence, causes and treatment of high cardiovascular risk: findings from the FINRISK Survey. Eur f Prev Cardiol 2012;19:1153-60.

[6] Lloyd-Jones D, Hong Y, Labarthe D, et al. Defining and setting national goals for cardiovascular health promotion and disease reduction: the American Heart Association's strategic impact goal through 2020 and beyond. Circulation 2010;121:586-613.

[7] Mozaffarian D, Benjamin E, Go A, et al. on behalf of the American Heart Association Statistics Committee and Stroke Statistics Subcommittee. Heart disease and stroke statistics-2015 update. A report from the American Heart Association. Circulation 2015;131:e29-322.

[8] Peltonen M, Laatikainen T, Borodulin K, et al. Prevalence of ideal cardiovascular health in an adult Finnish population: the national FINRISK 2007 study. Int HeartVasc Dis $\mathcal{f}$ 2014;2:3-10.

[9] Vartiainen E, Laatikainen T, Peltonen M, et al. Thirty-fiveyear trends in cardiovascular risk factors in Finland. Int $\mathcal{F}$ Epidemiol. 2010;39:504-18.

[10] Albus C. Psychological and social factors in coronary heart disease. Ann Med 2010;42:487-94.

[11] Perk J, De Backer G, Gohlke H, et al. European Guidelines on Cardiovascular Disease Prevention in Clinical Practice (version 2012). The Fifth Joint Task Force of the European Society of Cardiology and other societies on cardiovascular disease prevention in clinical practice. Eur Heart $\mathcal{F}$ 2012;33:1635-701. 
[12] Selvin E, Crainiceanu C, Brancati F, et al. Short-term variability in measures of glycemia and implications for the classification of diabetes. Arch Intern Med 2007;167:1545-51.

[13] The International Expert Committee. International Expert Committee report on the role of the A1c assay in the diagnosis of diabetes. Diabetes Care 2009;32:1327-34.

[14] Espana-Romero V, Artero E, Lee D, et al. A prospective study of ideal cardiovascular health and depressive symptoms. Psychosomatics 2013;54:525-35.

[15] Gaye B, Prugger C, Perier MC, et al. High level of depressive symptoms as a barrier to reach an ideal cardiovascular health. The Paris Prospective Study III. Sci Rep 2016;6:18951.

[16] Bush K, Kivlahan D, McDonell M, et al. The AUDIT alcohol consumption questions (AUDIT-C): an effective brief screening test for problem drinking. Ambulatory Car Quality Improvement Project (ACQUIP). Alcohol use disorders identification test. Arch Inter Med 1998;158:1789-95.

[17] Graciani A, Leon-Munoz L, Guallar-Castillon P, et al. Cardiovascular health in a southern Mediterranean European country: a nationwide population-based study. Circ Cardiovasc Qual Outcomes 2013;6:90-8.

[18] Jankovic S, Stojisavljevic D, Jankovic J, et al. Association of socioeconomic status measured by education and cardiovascular health: a population-based cross-sectional study, $B M F$ Open 2014;4:e005222.

[19] Olsen G, Holm A, Jorgensen T, et al. Distribution of ideal cardiovascular health by educational levels from 1978 to 2006: a time trend study from the capital region of Denmark. Eur F Prev Cardiol 2014;21:1145-52.

[20] Caleyachetty R, Echouffo-Tcheugui J, Muennig P, et al. Association between cumulative social risk and ideal cardiovascular health in US adults: NHANES 1999-2006. Int $\mathcal{7}$ Cardiol 2015;191:296-300.
[21] Zeng Q, Dong S, Song Z, et al. Ideal cardiovascular health in Chinese urban population. Int $\mathcal{F}$ Cardiol 2013;167: 2311-17.

[22] Cohen B, Edmondson D and Kronish I. State of the art review: depression, stress, anxiety, and cardiovascular disease. Am F Hypertens 2015;28:1295-302.

[23] Steptoe A and Kivimäki M. Stress and cardiovascular disease: an update on current knowledge. Anпи Rev Public Health 2013;34:337-54.

[24] Shim R, Baltrus P, Ye J, et al. Prevalence, treatment, and control of depressive symptoms in the United States: results from the National Health and Nutrition Examination Survey (NHANES), 2005-2008. f Am Board Fam Med $2011 ; 24: 33-8$

[25] Kronish I, Carson A, Davidson K, et al. Depressive symptoms and cardiovascular health by the American Heart Association's definition in the Reasons for Geographic and Racial Differences in Stroke (REGARDS) study. PloS One 2012;7:e52771.

[26] Wittchen H, Jacobi F, Rehm J, et al. The size and burden of mental disorders and other disorders of the brain in Europe 2010. Eur Neuropsychopharmacol 2011;21: 655-79.

[27] O’Dell K, Masters K, Spielmans G, et al. Does type-D personality predict outcomes among patients with cardiovascular disease? A meta-analytic review. F Psychosom Res 2011;71:199-206.

[28] Nielsen K, Faergeman O, Larsen M, et al. Danish singles have a twofold risk of acute coronary syndrome: data from a cohort of 138290 persons. F Epidemiol Community Health 2006;60:721-8.

[29] Whooley M. Screening for depression-a tale of two questions. $7 A M A$ Intern Med 2016;176(4):436-8. 